Geneviève Mottet

Université de Genève (Suisse)

Margarita Sanchez-Mazas

Université de Genève (Suisse)

\section{La formation initiale et continue \\ des enseignant-e-s à l'interculturel : discontinuités et injonctions paradoxales}

\author{
The initial and continuous training of teachers \\ in interculturality: discontinuities and paradoxical injunctions
}

doi: $10.18162 / f p .2021 .576$

\section{ésumé}

En s'intéressant au développement de la formation à l'interculturel au moment de l'académisation de la formation des enseignants à la fin des années 1990, cet article prend en compte tant la formation initiale dispensée

à l'université que la formation continue (catalogue de

formation à choix et perfectionnement obligatoire)

portée par l'autorité scolaire cantonale. Nous proposons d'examiner ces deux domaines de formation en retraçant le développement de la formation interculturelle du personnel enseignant, telle qu'elle s'est développée à Genève au cours des trente dernières années. À l'aide de données d'enquête

et d'une étude lexicale, nous interrogeons la cohérence d'ensemble de la formation des enseignants à l'interculturel et illustrons les particularités de milieux de formation aux objectifs parfois dissonants.

Mots-clés

Formation initiale des enseignants, formation continue, éducation interculturelle, diversité à l'école, catégorisations culturelles, enjeux éducatifs

\section{Abstract}

By focusing on the development of intercultural training during its process of integration within the academic system in the late 1990s, the present article takes into account the basic teachers' education at the university as well as continuing training (catalog of optional courses and mandatory professional development) carried out by the cantonal education authority. We propose to examine these two areas of teachers' education by tracing back the development of intercultural training, as it has evolved

in Geneva over the last thirty years. Using survey data and a lexical study, we examine the overall coherence of teachers' training in intercultural education and illustrate the particularities of training environments often pursuing divergent objectives.

Keywords

Basic teachers' education, continuing training, intercultural education, diversity in schools, migrant pupils, cultural categorizations, education issues

\section{Introduction}

La question de la formation des enseignants à la diversité ethnoculturelle est en interrelation étroite avec les transformations des contextes politiques et migratoires, celles des mots d'ordre internationaux et celles des investigations et recommandations des chercheurs. Les axes de formation et les thèmes mobilisés peuvent varier dans leur appréhension ou émerger selon les contextes sociohistoriques. En Suisse, la pédagogie interculturelle, après avoir connu une perte d'intérêt, connaît un nouvel essor dans les années 2010, remobilisée dans une visée de pédagogie générale (promotion des compétences interculturelles, pédagogie de la littéracie, du français langue seconde, de la pédagogie inclusive) comme un outil commun d'amélioration des compétences de tous (Mottet, 2017a). De fait, le réinvestissement de la pédagogie interculturelle (dans son sens large) par les politiques éducatives implique des modifications dans la manière de penser la formation des enseignants.

Depuis les attentats de 2015, de nouveaux thèmes liés à ces contextes politiques en forte tension ont également été intégrés à la formation des enseignants, alors qu'on observe de nouvelles formes de problématisation portant sur des élèves issus de la migration et notamment ceux d'origine musulmane (Mabilon-Bonfils, 2015; Mottet,2017b). L'institution scolaire entend former les professionnels et les élèves sur de nouvelles dimensions de la question migratoire à l'école. Par exemple, l'enseignement des faits religieux, la citoyenneté, ou la formation des enseignants à la détection de la radicalisation deviennent de nouveaux enjeux de formation. En ce sens, Zoïa (2013) rend compte du développement de la question laïque à l'école en France qui s'opérationnalise par de nouveaux enseignements pour les élèves, relevant de l'instruction civique et morale. Cette éducation 
apparaît aujourd'hui comme un «remède, une réponse, à des problèmes d'insécurité, d'autorité, de communautarisme...» (p.75) et est soutenue tant par l'extrême droite que par la gauche. Ces nouveaux objets de formation se développent actuellement en Suisse, comme dans beaucoup de pays, dans une visée qui se veut préventive, sécuritaire et/ou humaniste, invoquant notamment la question religieuse et la laïcité.

À partir du modèle genevois de formation des enseignants de niveau primaire, cet article s'intéresse à la manière dont l'intégration de la diversité culturelle a été pensée dans une haute école comme objet d'enseignement pour les futurs acteurs de terrain. Il cherche à rendre compte dans un premier temps de la constitution de ce nouvel axe de formation dans les années 1990 et du fait qu'il s'est réalisé sur le plan académique dans une certaine proximité avec le terrain. Il propose d'examiner, dans un second temps, le décalage qui se fait voir à la fin des années 2010 concernant le rapport à la diversité culturelle dans la formation entre le milieu académique et le terrain. Les nouveaux professionnels se trouventils face à une continuité entre les objectifs et messages délivrés par leurs formateurs universitaires lors de la formation initiale (FI) qu'ils ont suivie et ceux de l'institution scolaire qu'ils reçoivent une fois engagés, notamment par le biais des formations continues (FC), des directives que celle-ci transmet et des pratiques effectives des enseignants? Nous proposons ainsi d'examiner le rapport actuellement existant entre ces deux volets de la formation des enseignants dans le domaine de la diversité. Notre intention est de nous interroger sur leur complémentarité, voire leur compatibilité, et de mettre au jour les possibles inconsistances que le manque relatif d'articulation entre ces deux voies de formation pourrait laisser supposer. Dans un troisième temps, le propos visera à examiner des pistes d'action qui pourraient mieux articuler les formations initiales et continues, ainsi que les préoccupations du milieu académique avec celles des politiques éducatives et du terrain. Au fil de cet article, nous nous intéresserons aux différentes réflexions, constats et propositions de chercheurs francophones travaillant sur les enjeux de la formation à la diversité culturelle.

\section{Cadre théorique : la formation des enseignants à la diversité culturelle}

Des chercheurs ont montré comment, dès les années 1960, la question des enfants d'immigrés a été problématisée par les politiques éducatives, en même temps qu'elle est devenue un problème social (Hutmacher, 1987; Sayad, 2014; Mottet, 2017a). En Suisse, comme dans d'autres pays occidentaux, c'est avec le passage d'une immigration de travail à une immigration de peuplement dans la première moitié des années 1970 que l'État a été amené à se préoccuper des enfants de travailleurs migrants (Bolzman, Fibbi et Vial, 1996). Un tournant important s'est alors opéré au sein des autorités politiques dans la manière de concevoir la population immigrée non plus comme une force d'appoint, mais comme une population dont il fallait assurer l'intégration et la formation. Les enfants issus de la migration ont été petit à petit constitués en catégorie d'action publique (Mottet et Bolzman, 2009) et une politique éducative (recommandations, lois, structures) a été institutionnalisée à leur égard. Parallèlement, des dispositifs pédagogiques ont également vu le jour et, avec eux, la question de la formation des enseignants (Mottet, 2017a). Alors que ces interrogations ont été l'objet de réflexions de chercheurs depuis les années 1980 (Lorcerie, 1983; Alleman-Ghionda et Lusso-Cesari, 1986; Rey, 1986; Dasen, 1991; Ouellet, 1991; Perregaux, 1994) dans différents pays francophones, tels que la France, la Belgique ou le Québec, ainsi que dans des pays non francophones (Kerzil, 2002), les modalités de formation à promouvoir restent encore actuellement l'objet d'un vaste chantier.

2 - Formation et profession 29(1), 2021 
Aujourd'hui, un consensus international semble se dégager quant à la nécessité de développer chez les membres du personnel scolaire des compétences antidiscriminatoires et inclusives (Allan,2010; Unesco, 2015; Tutiaux-Guillon et Verhoeven, 2018). Potvin et Larochelle-Audet (2016) relèvent que parmi les écrits internationaux consultés, les auteurs des différents courants se rejoignent sur l'importance de développer pour le personnel enseignant une compréhension sociopolitique et historicisée des inégalités sociales et des systèmes d'oppression. Larochelle, Borri-Anadon et Potvin (2016) relèvent que pour ces auteurs, il s'agirait également de faire figurer dans le curriculum de base le développement de connaissances relatives aux multiples réalités et parcours des élèves issus de l'immigration et appartenant à des groupes minorisés, de même que celles permettant de mieux comprendre les réalités de leurs familles. Sanchez-Mazas, Mechi et Buchs (2021) mettent en exergue l'importance de former le corps enseignant à déterminer les effets de contexte et les dynamiques situationnelles dans les attitudes et les comportements autant des enseignants que des élèves, en particulier en contexte scolaire hétérogène. Pour ces auteures, ce type d'analyse gagne en efficience formative lorsqu'il porte sur des situations concrètes auxquelles les futurs professionnels ont participé ou assisté et qui sont, comme toute situation vécue, imprégnées d'affects et donc propices à toutes sortes de biais. En rupture avec une analyse en termes d'attributs des groupes ou de facteurs de personnalité, cette approche vise à comprendre les enjeux et les dynamiques propres à des situations comparables plutôt que des recettes à appliquer au coup par coup ou alors de manière indistincte. Santerini (2002) ajoute qu'il ne s'agit pas de considérer les seules attitudes et les comportements des individus sur le plan psychosocial, mais le contexte historique, social et civique dans lequel se révèlent les phénomènes collectifs. De plus, l'auteure prend en considération l'importance de développer des formations à l'interculturel non seulement spécifiques, mais également transversales à l'intérieur du cursus scolaire.

Alors que les recherches continuent de montrer que les jeunes issus de la migration, qu'ils soient primo-arrivants ou de migrations plus anciennes, connaissent toujours d'importantes inégalités d'orientation ou de réussite scolaires (Stevens et Dworkin, 2019; Gomensoro et Bolzman, 2016), il demeure très délicat d'aborder de front le problème des discriminations effectives avec les acteurs scolaires. Le numéro "Former contre les discriminations (ethno)culturelles» de la revue Recherche E Formation, coordonnée par Tutiaux-Guillon et Verhoeven (2018), met en exergue les difficultés relatives à la conscientisation des phénomènes d'inégalités et de discriminations dans les formations des enseignants, mais également et surtout celle d'amener les professionnels à changer concrètement leurs pratiques. L'article de Borri-Anadon, Collins et Boisvert (2018) rend compte de la difficulté qu'éprouvent les acteurs scolaires à procéder à des adaptations des contenus et des outils didactiques alors même qu'ils savent que certains d'entre eux et certaines de leurs pratiques revêtent un caractère potentiellement discriminatoire. De même, si dans le contexte de formation, et notamment lors de situations d'observations en stages, les étudiants se montrent capables d'identifier les actes et attitudes discriminatoires des professionnels (Jacobs, 2018; Capitanescu Benetti et Mottet, 2020), il n'en demeure pas moins que le passage de la conscientisation à la modification des pratiques reste encore un large chantier pour les formateurs du milieu académique. Différentes pistes sont pourtant travaillées dans le cadre des formations telles que celle proposée par Mechi et Sanchez-Mazas (2018) visant à déplacer l'accent souvent opéré sur l'individu et son appartenance vers la situation éducative et la possibilité de la modifier par le développement d'une flexibilité sociocognitive. 
Les recherches actuelles vont en tout cas dans le sens de la promotion dans les formations d'une distanciation relativement aux catégories d'attribution associées aux origines des élèves, les chercheurs relevant le risque de folklorisation et d'essentialisation (Abdallah-Pretceille, 1999; Larochelle-Audet, Borri-Anadon et Potvin, 2016).

Le paradigme dominant qui traverse désormais les instituts universitaires (ou les hautes écoles) est de former un «praticien réflexif» (Schön, 1994) où la capacité à interroger la situation et à s'interroger est privilégiée par rapport à l'application de «recettes préfabriquées», qui risquent de laisser les enseignants démunis face à la complexité des phénomènes en jeu (Troncin, 2011).

À ce sujet, peu de recherches analysent les différences entre formation initiale et continue. Plumelle et Latour (2012) ont bien réalisé une analyse comparative entre formation initiale et continue des enseignants en Allemagne, Angleterre et Finlande, mais sans explorer spécifiquement la question de la formation à l'interculturel. Besençon (2015) a effectué des comparaisons entres les formations initiales et continues en Suisse romande. Il présente les variations des politiques cantonales en relevant le fait que la formation continue est soit dispensée au sein des hautes écoles sous l'égide du milieu académique (hautes écoles), soit sur le terrain sous l'égide de l'institution scolaire. Il observe qu'au-delà des diversités locales, les départements de l'instruction publique des cantons exigent souvent que la mise en œuvre d'une nouvelle réglementation, d'un plan d'études ou d'un moyen d'enseignement soit accompagnée de cours obligatoires de formation continue et constate qu'a contrario il est très rare que la participation des professionnels soit formellement requise à des cycles de conférences présentant des comptes rendus de recherche mettant en évidence des connaissances nouvelles.

En ce qui concerne le thème de la diversité culturelle, nous avons relevé peu de recherches qui interrogent les différences entre formation initiale et continue. Nous pouvons nous référer à l'étude d'AllemanGhionda, De Goumoëns et Perregaux (1999) (déjà un peu ancienne) qui a évalué dans quelle mesure la dimension de la pluralité culturelle et linguistique était alors intégrée dans les programmes de formation initiale et quelles étaient les offres de formation continue proposées. Les auteures ont alors observé que dans les treize instituts de formation étudiés, la dimension de la pluralité culturelle et linguistique était uniquement associée à la question de la migration, principalement du point de vue des problèmes qui en découlent, et que l'éducation à la pluralité était rarement évoquée. À la suite de cette recherche, Lanfranchi, Perregaux et Thommen (2000) ont proposé dans leur rapport final des recommandations concernant l'officialisation d'un curriculum de formation visant à intégrer véritablement la pédagogie interculturelle dans les programmes de formation. De même, elles recommandent la mise en place de formations continues permettant de prolonger l'acquisition des compétences qui ne peuvent être toutes acquises durant la formation initiale.

Plus récemment, une recherche a également été menée en ce sens au Québec. Potvin et al. (20122015) ont effectué une étude sur l'enseignement de la diversité ethnoculturelle en formation initiale et continue des professionnels de l'éducation dans les universités. Elle visait à cartographier la formation du personnel scolaire sur la diversité et a montré l'expansion significative de ce champ depuis une dizaine d'années, tout en relevant les limites de cette offre de formation, telles qu'un faible ancrage institutionnel, une absence d'exigences et d'orientations ministérielles claires et des disparités entre régions et programmes (dans Borri-Anadon et al., 2018, p.8). Dans ce rapport, Borri-Anadon et al. (2018) réaffirment l'importance de rendre ces formations obligatoires pour l'ensemble du personnel 
éducatif en ce qui concerne la formation initiale, mais également la formation continue. Ce qui se fait effectivement dans les formations sur la diversité (ethno)culturelle reste difficile à établir, tant les dispositifs et pratiques se développent différemment selon les régions d'un même pays, selon les établissements, également selon les pratiques des formateurs et les terminologies employées. Si des tendances se dessinent nettement sur le plan de la formation initiale, notamment par le biais des réseaux qui se tissent entre chercheurs des différents pays (Potvin, Dhume, Verhoeven et Ogay, 2018), la globalité des offres de cours et des pratiques de formation réellement effectives reste difficile à appréhender.

Dans cette perspective, nous cherchons à apporter ici une contribution à la compréhension du processus de transformation des formations à la diversité culturelle et à la visibilisation de certains enjeux, problèmes et apports existants, à partir du cas circonscrit de la formation des acteurs scolaires dans le canton de Genève.

\section{Une méthodologie plurielle pour appréhender les formations à l'interculturel}

Nous nous appuierons sur une analyse de sources écrites et orales variées qui n'ont pas le même statut empirique selon les thématiques abordées. En premier lieu, concernant le déploiement de la formation initiale à l'interculturel à Genève, nous étayons nos observations sur les données récoltées dans le cadre d'une enquête (Mottet, 2013, 2017a) portant sur les dispositifs promus entre les années 1960 et aujourd'hui dans le canton (ainsi qu'au niveau national) en faveur des élèves d'origine étrangère. Ces sources consistent en une trentaine de documents émanant d'acteurs institutionnels et/ou de chercheurs locaux (du canton de Genève) et d'entretiens compréhensifs approfondis menés auprès de deux d'entre eux. Elles rendent compte des «premiers pas» de la pédagogie interculturelle (création de moyens pédagogiques et formation des acteurs professionnels) dans le contexte d'une académisation de la formation des enseignants au milieu des années 1990.

En second lieu, pour documenter la formation actuelle des enseignants à l'interculturel, les sources analysées proviennent de la recension des enseignements académiques (formation initiale) au sein de la Faculté des sciences de l'éducation (FPSE) et de ceux donnés sous l'égide de l'autorité scolaire cantonale (formation continue) sur ce thème. Nous avons cherché à confronter les intitulés et les descriptifs des programmes de formation initiale (FI) et continue (FC) concernant l'année académique (2017-2018) ${ }^{1}$. Une analyse discursive de la fréquence des mots clés figurant dans les catalogues - à savoir, d'une part, le Guide/programme de la Section des sciences de l'éducation (2017-2018) (FI) et d'autre part, le Catalogue de formation de l'enseignement primaire (2017-2018) (FC) - a été réalisée au moyen du logiciel N’Vivo. Concrètement, nous avons extrait une vingtaine de mots clés, ceux les plus fréquemment mobilisés dans les descriptifs de ces cours, puis nous avons calculé leur fréquence en pourcentage et les avons regroupés en thématiques. La question était de savoir, si, en matière des transformations évoquées, les approches formatives privilégiées tendent à converger ou à diverger entre les sphères académique et institutionnelle.

En troisième lieu, selon une approche qui se veut exploratoire, nous effectuons des propositions émanant de la littérature et des expériences professionnelles quant aux modalités, rythmes et temporalités sur le plan de la formation. Lobjectif sera alors de réfléchir à une plus grande perméabilité entre les préoccupations des acteurs du terrain ou des responsables des politiques éducatives et celles des acteurs du milieu académique (université ou hautes écoles). 


\section{Contexte : la formation des enseignants à la diversité à Genève}

Avec la déclaration de Bologne, la Suisse, comme d'autres pays européens, a connu une redéfinition des frontières entre formations professionnelles et formations académiques. Cela s'est traduit par la création des Hautes écoles spécialisées (HES) à la fin des années 1990 et par l'intégration à l'université de certains domaines de formation qui relevaient auparavant de filières professionnelles. Concernant la formation des enseignants du primaire, la plupart des cantons suisses ont opté pour la création des Hautes écoles pédagogiques (HEP) remplaçant les différentes formations cantonales antérieures, tandis que le canton de Genève a intégré celle-ci à l'université en 1997.

Les premiers pas d'une politique d'intégration (ouverture aux étrangers, promotion de leur participation et lutte contre les discriminations) et l'académisation de la formation des enseignants au milieu des années 1990 ont eu un effet sur les possibilités de déploiement de la formation à l'interculturel, d'autant que la constitution du domaine à l'université a bénéficié au départ de l'engagement d'acteurs de terrain, aussi bien à l'échelle des instances supranationales qu'à l'échelle locale. L'investissement de certains experts genevois, comme Micheline Rey ou Christiane Perregaux, au sein d'organisations telles que l'UNESCO ou le Conseil de l'Europe, a eu des effets sur le développement particulièrement important dans ce canton, tant de la recherche que de la formation des enseignants et des moyens pédagogiques concernant la dimension interculturelle de l'éducation.

Depuis le début de l'académisation de la formation des enseignants primaires, à la fin des années 1990, entre sept et dix cours par an s'inscrivent explicitement au sein de la formation initiale (FI) ${ }^{2}$ dans le cadre de la formation interculturelle et de la prise en compte des élèves d'origine étrangère. En ce qui concerne le catalogue de formation continue $(\mathrm{FC})^{3}$ pour les enseignants du primaire genevois, quinze offres de cours sur ces thèmes ont été faites pour l'année 2017-2018. En outre, deux cours de perfectionnement obligatoires ont été mis en place par l'autorité scolaire en 2018 sur les thèmes de la prévention de la radicalisation et de l'enseignement du fait religieux. Relevons que si le nombre de cours dans les deux systèmes de formation peut être quantitativement comparable, ce n'est pas le cas pour ce qui est du nombre d'heures allouées par les formations puisque le milieu académique (FI) offre plus d'heures de formation (cours semestriels) que le département de l'instruction publique (formations ponctuelles pour la FC). En outre, le caractère obligatoire ou motivé des formations varie également selon le contexte et les offres de formation.

\section{Naissance et académisation de la formation à l'interculturel}

À Genève, la formation à l'interculturel a été institutionnalisée dans la formation des enseignants dans le contexte de son académisation. L'analyse propose ici de mettre en exergue ce développement à partir de discours et pratiques d'experts locaux, investis parallèlement dans le Conseil de l'Europe.

Selon Rey (1996), le groupe d'experts du Conseil de l'Europe, dont elle faisait partie, a réussi à «introduire l'interculturel, susciter une réflexion autour de la notion d'éducation interculturelle, contribuer à sa conceptualisation, à sa diffusion et à sa mise en pratique dans les différents pays d'Europe, dont la Suisse»(p.44). L'extrait d'entretien de Perregaux, professeure-chercheuse pour la formation interculturelle des enseignants dans le cadre de l'académisation de la formation, rend

6 - Formation et profession 29(1), 2021 
compte de la fabrique de l'éducation interculturelle au niveau cantonal, national et international (Mottet, 2017a). Perregaux se réfère aux premières recherches publiées sur la question, ainsi qu'au travail de mobilisation de Rey qui a été enseignante au cycle d'orientation ${ }^{4}$ à Genève puis chargée de cours à l'université et qui a participé à la mise en place des premières formations à l'interculturel en milieu universitaire.

Si vous prenez Genève, si vous prenez la Suisse, avec toute la littérature qui a commencé à paraître sur l'éducation interculturelle. Avec les travaux du Conseil de l'Europe, puis d'autres travaux pour prendre l'aspect plus francophone, il y a eu une littérature qui a commencé à se mettre en place. Ici à Genève, il y a eu Micheline Rey qui a beaucoup travaillé au Conseil de l'Europe et qui a été une personne ayant servi de catalyseur (Entretien, Christiane Perregaux, Professeure honoraire en Sciences de l'éducation).

L'académisation de l'ensemble de la formation primaire s'est faite également sous l'égide de Perregaux, professeure-chercheuse issue du terrain, ayant acquis une expertise à la fois académique et dans les instances supranationales en matière de formation interculturelle. Cette convergence entre implication internationale d'acteurs de terrain, arrimage de la formation à la recherche en éducation et volonté politique des milieux décisionnels cantonaux a contribué à faire de Genève un lieu pionnier dans la réflexion et dans le déploiement de la formation à l'interculturel au cours des années 1990.

C'est dans ce contexte de reconnaissance de l'impératif de former les enseignants à l'interculturalité que le besoin de produire des ressources pédagogiques appropriées s'est fait sentir. Pour Perregaux, l'idée est de «trouver quelque chose à mettre à disposition des enseignants» (Entretien, Christiane Perregaux). De plus, progressivement, les premiers outils pédagogiques et méthodologiques relatifs à l'éducation interculturelle ont commencé à être élaborés dans l'objectif d'ouvrir le regard des enfants à la diversité des langues et de favoriser l'ouverture à l'Autre (Alleman-Ghionda et al., 1999; Candelier 2003; Perregaux et al., 2003).

La formation à l'interculturel s'est déployée en phase avec les priorités de l'autorité scolaire du moment. De fait, le département de l'instruction publique a promu la constitution d'un groupe de travail auquel ont collaboré des chercheurs universitaires et des acteurs institutionnels, sous l'égide de l'autorité cantonale. Comme l'explicite une des personnes interviewées, anciennement responsable du Service des élèves non francophones (SENOF) du département de l'instruction publique, son service a travaillé en étroite collaboration avec l'université au moment de l'institutionnalisation de la formation du personnel enseignant dans la section des sciences de l'éducation. Les propos rendent compte de l'émulation qui a été permise dans ce contexte d'innovation, qui a favorisé le développement d'une formation des enseignants à l'interculturel par la collaboration entre les différents acteurs du milieu académique et de l'institution scolaire.

Je faisais partie des commissions de la pédagogie, où l'interculturel avait une place [...] et du groupe de réflexion pour la formation des enseignants à l'université. [...] C'est un virage. Et là, nous avons travaillé avec Christiane Perregaux, Pierre Dasen etc. Dès la première année, il y avait une bonne place accordée à l'interculturel et nous avons dès ce moment-là, travaillé en étroite collaboration avec le service de pédagogie générale et la FAPSE, d'autant que Christiane Perregaux est une maitresse enfantine que nous connaissions... Donc, il y a eu beaucoup de travail qui a été fait au plan linguistique : ressemblances, différences, parentés dans les langues latines... On a fait un très gros travail (Responsable du SENOF, cité dans Mottet, 2013, p. 231). 
Le développement du domaine de l'interculturel à l'université, y compris lorsqu'il s'est élargi au début des années 2000 à l'enseignement secondaire et spécialisé, a bénéficié de la liberté académique qui a laissé aux professeur-e-s une marge considérable dans le développement des contenus, des thèmes de recherche et des méthodes pédagogiques. Il en a résulté des thématisations du domaine évoluant au gré des expertises et intérêts des spécialistes en poste et des exigences changeantes du terrain. Devant l'expansion naissante du champ de l'enseignement à la diversité interculturelle, les offres de formation vont ainsi se diversifier, sur le plan tant de la formation initiale que de la formation continue, à Genève, comme d'autres cantons ou pays francophones. Dans ces contextes de développement d'un tel objet de formation, certaines limites telles que l'absence d'exigences et d'orientations ministérielles claires et des disparités entre régions et programmes (Borri-Anadon et al., 2018) sont à questionner, ainsi qu'un manque de concertation entre des spécialistes, plus nombreux, qui participent d'une étape ou d'une autre de la formation (initiale et continue).

\section{Des projets divergents entre la formation initiale (FI) et la formation continue $(\mathrm{FC})$ à l'interculturel?}

Dans la mesure où la thématique de l'interculturalité est devenue parallèlement l'objet de différentes formations continues pour les enseignants en fonction, nous avons tenté, à la suite de Borri-Anadon et al. (2018), de comparer et d'analyser le développement qui s'est déployé corrélativement dans ces deux contextes de formation (FI et FC), dans le contexte circonscrit du canton de Genève.

Alors que, comme nous l'avons vu, une collaboration entre responsables de l'autorité scolaire et chercheurs du milieu académique a été réalisée dans les années 1990, il s'agit à présent de se pencher sur le rapport existant actuellement en matière des formations à l'interculturel entre milieu académique et autorité scolaire. La mise en regard des intitulés et résumés des formations données dans les deux contextes pendant l'année scolaire 2017-2018 nous fournira des indications.

À ce titre, ainsi qu'il ressort du catalogue 2017-2018 de formation continue de l'enseignement primaire (FC) concernant les élèves issus de la migration, peu de propositions de cours émanent de personnes du milieu académique (université, HEP). Les experts sollicités pour dispenser des formations proviennent essentiellement du terrain (enseignant-e-s du primaire ou secondaire, doyens) et présentent souvent une spécialisation professionnelle (psychologue, interprète, infirmière spécialisée). Ce constat rend compte d'une différence d'inscription professionnelle des formateurs selon les contextes de formation et du déploiement probable d'une pluralité d'approches et de terminologies mobilisées (Borri-Anadon et al.,2018). Certainement, la question se pose de savoir pour quelles raisons les formateurs responsables de la formation initiale proposent rarement des formations dans le catalogue pour les enseignants en fonction $^{5}$. Si le manque de sollicitation de la part de l'institution scolaire peut être une raison, d'autres peuvent également s'exercer comme le caractère non obligatoire de cet investissement dans les cahiers des charges des formateurs universitaires, le manque de temps par rapport aux différentes tâches à mener ou encore la moindre reconnaissance de ce travail par rapport à l'attente institutionnelle de recherche et de publications. Une autre hypothèse pourrait être que des formateurs universitaires dans le champ de l'éducation à la diversité ethnoculturelle considéreraient les difficultés relatives à la conscientisation des phénomènes d'inégalités et de discriminations dans les formations des enseignants en fonction 
(Tutiaux-Guillon et Verhoeven, 2018). Ils éviteraient ainsi de se trouver face à un terrain qui, selon eux, rechercherait avant tout des recettes toutes faites (Troncin, 2011).

Cependant, au-delà des questions d'expertise des intervenants, il s'agit d'interroger les tendances qui se dessinent selon les contenus de formation à la diversité culturelle proposés à chacun des niveaux sur la base d'une comparaison lexicale des contenus des catalogues (ou guides) de formation (FI et FC). Comme présenté dans la méthodologie, nous avons extrait une vingtaine de mots clés, ceux les plus fréquemment mobilisés dans les descriptifs de ces cours, puis nous avons calculé leur fréquence en pourcentage et les avons regroupés en thématiques (cf. tableau 1). Certaines discordances peuvent être observées entre les programmes de formation initiale en milieu universitaire pour l'année académique 2017-2018 et ceux proposés parallèlement par l'autorité scolaire aux professionnels de terrain en emploi. Des différences apparaissent entre les intitulés et mots clés figurant dans les descriptifs des cours. En ce qui concerne la FI en milieu académique, nous avons recensé dix cours qui s'inscrivent dans notre thématique, et seize pour la FC proposés par le département de l'instruction publique. Nous voyons ci-dessous les termes les plus mobilisés (deuxième colonne) que nous avons regroupés par catégories (première colonne). Les pourcentages rendent compte des fréquences de l'usage de ces termes dans chacune des présentations.

\section{Tableau 1}

Fréquence des catégories des mots tirés des catalogues de formation à l'interculturel en formation initiale et continue à Genève en 2017-2018 (exprimées en \%)

\begin{tabular}{|c|c|c|c|}
\hline Catégories & Mots (termes les plus mobilisés) & $\begin{array}{c}\text { FC } \\
\text { catalogue } \\
\text { DIP }\end{array}$ & $\begin{array}{l}\mathrm{Fl} \\
\text { catalogue } \\
\text { université }\end{array}$ \\
\hline Apprentissage scolaire (discipline, etc.) & $\begin{array}{l}\text { langue, linguistique } \\
\text { apprendre, apprentissage } \\
\text { formation } \\
\text { français } \\
\text { disciplines, domaines } \\
\text { objectifs } \\
\text { enseignement } \\
\text { écrit } \\
\text { production }\end{array}$ & 33.5 & 2.2 \\
\hline Outils, pistes & perspectives, pistes, pratiques & 17.5 & 3.8 \\
\hline Diversité & différence, différent, diversité, pluralité hétérogène & 8.9 & 14.2 \\
\hline Culturel, interculturel & cultures, culturel, interculturel & 8.6 & 21.3 \\
\hline Contexte, situation & contexte, situation & 8.4 & 12.6 \\
\hline Catégorie (allophones, suisse, islam) & $\begin{array}{l}\text { allophone, élèves migrants, élèves érythréens, } \\
\text { arabophones, afghans } \\
\text { islam, suisse }\end{array}$ & 6.3 & 0 \\
\hline Compétence & compétences & 1.9 & 1.6 \\
\hline Difficultés & difficultés & 1.7 & 0 \\
\hline Analyses, dynamiques et enjeux & $\begin{array}{l}\text { analyse, analyser, concepts } \\
\text { dimensions, enjeux (rapports, relations) } \\
\text { dynamiques, mécanismes, processus }\end{array}$ & 0 & 20.8 \\
\hline Stéréotypes & stéréotypes & 0 & 2.7 \\
\hline
\end{tabular}


La comparaison met en lumière les contrastes dans la fréquence des mots utilisés. On observe en effet que les références aux disciplines scolaires et aux apprentissages sont très présentes dans le catalogue du DIP (FC), alors qu'elles sont quasiment absentes de celui de l'université (FI). Les mots "apprendre », "formation", "français", "disciplines", «objectifs", "écrit», etc. sont ainsi mobilisés à 33,5 \% dans le catalogue FC contre 2,2 \% dans les descriptifs des cours en formation initiale. De même, l'usage des termes relatifs à des outils ou des pratiques d'action ("perspectives», "pistes», "pratiques») est beaucoup plus le fait des projets de formation en emploi (FC) que de ceux de la formation initiale $(17,5 \%$ contre $3,8 \%)$. Il est également significatif que le terme interculturel soit plus fréquemment mobilisé par le milieu académique (21,3\% contre 8,6 \%). Ceci pourrait bien résulter d'une visée de ses formateurs de ne pas cibler des origines spécifiques (Larochelle-Audet, Borri-Anadon et Potvin, 2016), alors que certains formateurs engagés dans le cadre de la formation continue nomment leurs cours en faisant précisément référence aux origines spécifiques des élèves (exemples d'intitulés de cours en formation continue : «scolarisation des élèves érythréens", "scolarisation des élèves arabophones", «scolarisation des élèves afghans», "l'islam et les musulmans en Suisse»). On voit par ailleurs des références aux catégories telles qu' «allophone», «suisse» ou «islam» qui figurent parmi le nombre de mots mobilisés en formation continue et absents en formation initiale universitaire $(6,3 \%$ contre 0 $\%)$.

Ces observations pourraient refléter des orientations différentes de ces dispositifs de formation. Cette hypothèse pourrait être étayée par le constat d'une autre différence qui s'observe dans l'usage de mots comme "analyser», "concepts», «dimensions» ou "enjeux», «dynamiques ", "mécanismes» et "processus» selon les contextes de formation. On observe en ce sens une fréquence de 20,8\% des termes les plus utilisés dans les descriptifs des cours académiques sur ce champ sémantique contre $0 \%$ dans les cours de formation continue.

L'analyse montre néanmoins une certaine convergence en ce qui concerne l'usage de termes comme «compétences» $(1,9 \% \mathrm{FC}$ et 1,6 \% FI), «contextes et situations» $(8,4 \% \mathrm{FC}$ et $12,6 \% \mathrm{FI})$ ou encore le rapport à la diversité des publics $(8,9 \% \mathrm{FC}$ et $14,2 \% \mathrm{FI})$.

La figure 1 illustre les différences d'usage des catégories évoquées et donne une image de la manière d'aborder les contenus liés à la question migratoire dans le cadre des projets et des conceptions de formation.

Ainsi, pour ce qui est des perspectives adoptées en FI et FC, une opposition apparaît entre les savoirs conceptuels visant à éclairer les pratiques, tels que les mobilisent la FI, et des approches ciblant les connaissances de groupes spécifiques d'élèves étrangers, telles que les développent plus souvent les FC. Il semble donc que, tandis que les étudiants sont amenés à tenir compte des contextes sociopolitiques, historiques et des enjeux (Potvin et Larochelle-Audet, 2016), ainsi que des mécanismes et dynamiques à l'œuvre dans une situation éducative (Sanchez-Mazas, Mechi et Buchs, 2021), les intervenants recrutés par l'autorité scolaire forment plutôt les professionnels à intervenir auprès d'élèves catégorisés à l'aide de marqueurs d'appartenance. 


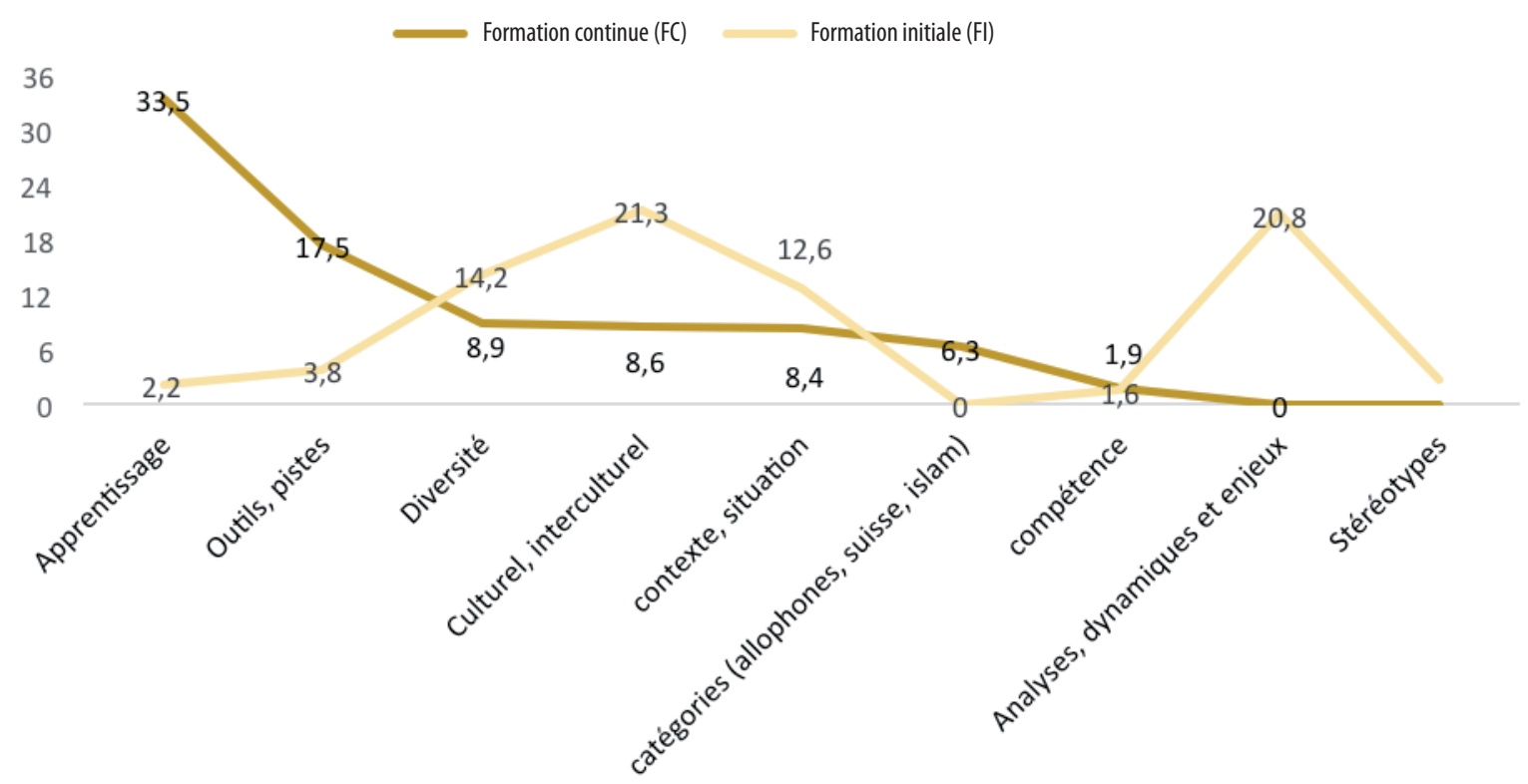

\section{Figure 1}

Usage des catégories des mots tirés des catalogues de formation à l'interculturel en formation initiale (FI) et continue (FC) à Genève en 2017-2018 (exprimées en pourcentages)

Cette comparaison entre indicateurs des formations à l'interculturel données en FI et en FC fait émerger un certain nombre de questions, telles que la manière dont s'élabore concrètement le choix des offres de formation. Pour autant, si l'on voit des tendances différentes entre FI et FC, cela ne signifie pas nécessairement que les formateurs de chaque institution nont pas collaboré ou échangé sur ces questions au moment de produire leurs dispositifs de formation et qu'ils n'adaptent pas leur offre au contexte de formation et au public présent. Dès lors, certaines limites de la démarche méritent dêtre ici mentionnées. Le travail sur les intitulés et descriptifs n'éclaire qu'imparfaitement ce qui se dit et s'enseigne concrètement et pratiquement dans le cadre de la formation et ne rend pas précisément compte de la mobilisation réelle des différentes catégories dans un contexte comme dans l'autre.

Il n'en demeure pas moins que l'ancrage institutionnel semble engager différemment les approches de la formation à l'interculturel et qu'une pensée globale et articulée de formation entre l'université et le terrain gagnerait à être promue. Si, comme nous l'avons vu, cette pensée globale a pu exister au moment des premières formations à l'interculturel, celle-ci pourrait bien sêtre progressivement diluée. Les décalages que nous avons relevés ici pourraient d'ailleurs s'accentuer dans la période récente, marquée par des inquiétudes croissantes du côté des politiques éducatives, dans le contexte d'une médiatisation du phénomène de radicalisation et des remous autour de l'islam.

En effet, si la question de la scolarisation des élèves affiliés à l'islam apparaît, comme nous l'avons vu, en filigrane dans les objets des FC, l'autorité scolaire a également décidé d'organiser un perfectionnement obligatoire (formation obligatoire dans le cadre de la FC) pour l'ensemble du corps enseignant de tous les degrés de scolarité sur le phénomène de la radicalisation (islamiste, mais également d'extrême droite). Lintention est que «tout le personnel qui côtoie au quotidien les élèves doit mieux reconnaître les signes avant-coureurs de la radicalisation» (Tribune de Genève du 30 août 2017)6. 
Selon la cheffe du DIP, il faut agir au niveau préventif en formant le personnel des écoles à «apprendre à repérer les élèves à risque, en étant attentif à un environnement familial et social fragilisé ou à des pratiques religieuses hyperritualisées» (Tribune de Genève, op. cit.).

Cet investissement massif sur la question de la radicalisation (plusieurs modules de formation dans différents ordres d'enseignement) peutêtre interrogé quant aux effets indésirables qu'il risque d'engendrer, pouvant participer à la mise en altérité des jeunes, notamment de ceux affiliés à la religion musulmane (Asal, 2014; Mabilon-Bonfis, 2015; Frisina, 2017; Mottet, 2017b), ainsi qu'à la stigmatisation et à la suspicion accrues envers certains publics, dans un contexte social où la xénophobie tend à se banaliser en dépit des dispositifs de lutte contre les discriminations.

Par ailleurs, de nouveaux moyens pédagogiques concernant l'enseignement du fait religieux et des batteries de FC obligatoires ont été introduits depuis la rentrée 2018 par le département de l'instruction publique à destination de l'ensemble des enseignants genevois ${ }^{7}$. Alors que le thème de la «laïcité» monte ainsi en puissance, les réponses politiques aux problèmes dénoncés cristallisent la question, notamment autour des pratiques professionnelles et des apprentissages à promouvoir ${ }^{8}$. Le débat qui se déploie ainsi sur l'enseignement du fait religieux dans une école laïque n'est pas sans lien avec la problématisation récente de l'islam dans le contexte scolaire et s'imbrique aux craintes de radicalisation des jeunes, notamment de ceux à risque de déscolarisation.

\section{Faire une cause commune de la formation à la diversité culturelle : besoins et pistes d'action}

Comment interpréter le décalage décelé entre FI et FC, notamment selon des approches qui semblent renvoyer d'une part à des situations éducatives problématiques et à la sensibilisation aux stéréotypes et d'autre part à des élèves problématiques en vertu de milieux socioculturels propices aux risques ? De même, quelles pratiques de formation pourrait-on développer afin que les professionnels ne se sentent pas tiraillés entre des normes et des approches pouvant s'avérer discordantes, voire paradoxales (Ogay et Edelmann, 2011) ?

Ces questionnements pourraient motiver le développement de recherches portant de manière approfondie sur les contenus d'enseignement relatifs à la diversité culturelle et leur réception par les professionnels, tout en prenant en compte leurs caractéristiques, expériences et trajectoires et le contexte du terrain dans lequel ils s'inscrivent. Une meilleure connaissance des représentations, résistances et arrangements qui s'opèrent chez les personnes formées (FI et FC) devrait donner des clefs de compréhension pour réfléchir à de nouvelles approches formatives. Il convient à cet égard de prendre en compte l'importance des aspects plus psychosociaux, relatifs aux différents milieux d'insertion des étudiants et des praticiens et des exigences et attentes propres à chacun des milieux. Il s'agirait d'étudier la transition des étudiants vers leur statut de professionnels et les injonctions que l'adaptation à la culture d'établissement, et plus largement, aux normes de la profession, font peser sur leur professionnalisation et leur acceptation par les collègues. Il se peut en effet que, pour ces raisons qui demeurent encore à investiguer, les jeunes enseignants soient amenés à prendre de la distance vis-à-vis des ancrages de la formation initiale, à adopter des perspectives qui en sont, au moins en partie, discordantes, et à adhérer à une certaine défiance formulée par les «praticiens » vis- 
à-vis des «académiques» en raison notamment de la réalité du terrain, voire de positions politiques ou idéologiques des enseignants ou des injonctions de l'autorité scolaire. Pour Borri-Anadon, Collins et Boisvert (2018), il est essentiel que les dispositifs de formation prennent davantage en compte les contraintes institutionnelles pour que la responsabilité de l'instauration de pratiques équitables et non discriminatoires ne repose pas que sur les épaules des professionnels.

Il importe également de bien se rendre compte que la formation dans le domaine de la discrimination est d'autant plus complexe que les obstacles tiennent paradoxalement à la prévalence d'une pensée «égalitariste» et d'une norme antidiscriminatoire, qui amènent la plupart des professionnels à se percevoir comme exempts de préjugés (Dhume, 2014). Dépasser les effets d'imperméabilité à une formation abordant la question des discriminations se heurte donc à des résistances de taille, d'autant plus que le préjugé revêt des formes de plus en plus subtiles (Sanchez-Mazas, 2004) et que la discrimination s'exerce souvent de manière indirecte dans les institutions (De Rudder et Vourc'h, 2006).

Devant ces premières observations, il s'avérerait pertinent d'envisager des pistes permettant de mieux articuler la formation théorique et pratique, de mieux dialoguer entre sphère académique et terrain, et par là de rendre plus opérante la formation à l'interculturel. Si celles-ci émergent, il est vrai, de recherches menées généralement par le milieu académique, elles peuvent néanmoins établir des ponts qui permettent de rejoindre les situations et les questionnements du terrain. De même, certains chercheurs encouragent le développement de recherches-actions, collaboratives ou participatives, envisageant des rapports horizontaux entre les formateurs du milieu académique et les professionnels de terrain. Le travail de coopération devrait pouvoir permettre de travailler sur les enjeux du terrain et de promouvoir le dialogue entre des acteurs ayant des connaissances différentes, mais complémentaires de la question de la diversité dans le champ scolaire.

De même, un travail de réflexion, soutenu par des liens institutionnalisés, entre formateurs du milieu académique et ceux du département de l'instruction publique serait particulièrement heuristique pour réfléchir en collaboration autour de la complexité de la formation à l'interculturel, de ses enjeux et des projets d'éducation souhaités. Une problématisation des focales distinctes proposées par les différents formateurs, soit sur le plan de l'approche par les catégories d'élèves soit à partir de situations éducatives complexes, constituerait un axe de réflexion partagée pertinent. Il s'agirait sans doute d'une piste pour réfléchir aux biais qui peuvent accompagner la centration sur des publics définis par leurs caractéristiques culturelles, même si celles-ci sont présentées de manière positive (richesse des langues d'origine, etc.).

De même, les collaborations entre contextes de formations et les réaménagements des modalités de formation classique pourraient être pertinents. Ainsi, Tessier et Le Roy (2009) proposent un modèle de formation à l'interculturel qui va à l'encontre de la logique récurrente d'une formation initiale «longue» suivie par des micro-enseignements de formation continue se succédant au cours de la carrière. Ils imaginent un modèle de «re-formation» construit autour d'un temps long de formation (de six mois) après une pratique professionnelle d'une ou deux années. Leur intention est de «réapprocher» l'interculturalité avec des regards plus «matures». À défaut de pouvoir tester ce modèle de formation (obstacles institutionnels), les auteurs proposent aux professionnels de terrain des formations d'une semaine entière. Selon les auteurs, cette intensité de formation permettrait ainsi 
aux acteurs de terrain de réinvestir les notions enseignées à la lumière de leur connaissance pratique et vécue du terrain, mais dans un temps suffisamment conséquent pour s'immerger et se confronter aux visées de formations académiques. Troncin (2011) met également en évidence la pertinence de construire un maillage cohérent des actions de formation, soit de faire des ponts institutionnalisés entre les différentes formations proposées. Il encourage la construction d'un maillage des actions de formation à la fois horizontalement, entre les divers dispositifs, et verticalement en développant une prise en compte de plus en plus élaborée de diversités de plus en plus complexes.

Ces tentatives de repenser la logique structurelle habituelle et la temporalité des formations à l'interculturel pour promouvoir des pratiques par lesquelles la formation initiale et celle en emploi s'imbriquent de manière innovante nous apparaissent être une piste intéressante à saisir.

\section{Conclusion}

Dans cet article, nous avons tout d'abord montré comment la formation à l'interculturel s'est déployée dans le contexte d'une académisation de la formation des enseignants alors que l'intérêt institutionnel porté sur cette question sociale relève d'enjeux politiques, économiques et éthiques du moment. Nous avons ensuite effectué une analyse comparative de deux contextes de formation du corps enseignant sur le rapport aux élèves issus de la migration et interrogé la concordance entre la formation initiale et le perfectionnement professionnel en cours d'emploi. Enfin, nous appuyant sur des propositions d'experts, nous avons tenté de proposer des pistes pour améliorer la cohérence des formations à la diversité et notamment sur le plan de l'articulation entre la formation initiale et continue.

Concernant nos observations des variations entre les formations données dans le milieu académique (FI) et en cours d'emploi (FC), il est apparu que celles-ci ne proposent pas le même type d'outillage. La FI promeut des outils encourageant la prise de distance face aux catégorisations stéréotypées, alors que la formation continue et le perfectionnement obligatoire portent en partie sur les spécificités groupales et le repérage des risques selon l'appartenance des élèves. Relevons bien évidemment que la formation continue des professionnels propose également des pistes de compréhension et d'action prenant en compte les situations d'enseignement. Or, nous pourrions avancer que ces pistes se veulent généralement plus pragmatiques qu'interprétatives des phénomènes et des enjeux en situation, à l'inverse de la formation initiale qui engage volontiers des pistes à partir d'une analyse contextuelle se centrant en priorité sur la compréhension des mécanismes et l'indépendance de la pensée en situation (Camilleri, 1990; Sanchez-Mazas, Changkakoti et Broyon, 2015).

Si notre analyse porte sur des offres de formation concernant l'année académique 2017-2018, il est possible que celles-ci aient depuis lors été quelque peu modifiées, et que des axes aient pu être ajoutés ou supprimés. Pour autant, le questionnement posé a permis de rendre compte d'enjeux, de besoins et de difficultés qui restent bel et bien d'actualité et dont il importe que les différents acteurs, qu'ils soient formateurs, acteurs de terrain ou politiques se préoccupent. Développer des recherches en variant les approches, promouvoir la collaboration et documenter les pratiques et les constats devraient sans doute contribuer à l'amélioration des formations à la diversité, au perfectionnement des pratiques professionnelles et à une plus-value de la scolarité des élèves issus de l'immigration qui, rappelons-le, restent sujets de pratiques discriminatoires. 
En outre, l'analyse et la réflexion menées ici à partir d'un contexte circonscrit à un canton suisse contribuent à l'avancement de la réflexion dans le champ de l'interculturel, notamment en ce qui concerne l'analyse de l'articulation existant entre formation initiale et continue qui est encore peu étudiée, alors qu'elle révèle des enjeux et des préoccupations au cœur des politiques éducatives. Nos questionnements et observations rejoignent également les interrogations et analyses de chercheurs travaillant sur d'autres contextes nationaux, notamment en ce qui concerne les pays francophones que sont le Québec, la France et la Belgique.

\section{Notes}

1 Nous n'avons pas intégré les cours de formation continue proposés dans le cadre de formations certifiantes comme des CAS (Certificate of Advanced Studies) ou DAS (Diploma of Advanced Studies).

2 Au niveau du bachelor (ou anciennement de la licence). Voir en annexe «Tableau a)» pour les intitulés de cours de FI.

3 Voir en annexe «Tableau b)» pour les intitulés de cours de FC.

4 Correspond au collège (entre 12 et 15 ans environ).

5 Ceux-ci sont parfois appelés par les écoles pour donner des formations continues à l'ensemble des enseignants d'un établissement. Nous n’avons pas intégré ces éléments, étant donné que nous n'avions pas accès à ces données et que nous souhaitions comparer les catalogues de formation officiels.

6 Borden, S.R. (2017, 30 août). Les enseignants vont être formés au phénomène de la radicalisation. Tribune de Genève, p. 15).

7 Cf. Armanios, R. (2018, 26 avril). L'accent mis sur les faits religieux. Le Courrier.

8 Pour la première fois dans le canton de Genève, en 2016, une brochure intitulée: La laïcité à l'école a été publiée par le département de l'instruction publique et distribuée à tous les enseignant-e-s (DIP, 2016).

\section{Références bibliographiques}

Abdallah-Pretceille M. (1999). Léducation interculturelle. Paris : Presses Universitaires de France.

Allan, J. (2010). Politiques et pratiques de l'enseignement de la diversité socioculturelle. Diversité et inclusion : enjeux pour la formation des enseignants. Strasbourg : Conseil de l'Europe.

Allemann-Ghionda, C., Perregaux, C. \& De Goumoens, C. (1999). Curriculum pour une formation des enseignant(e)s à la pluralité culturelle et linguistique. PNR 33. Berne et Aarau : Centre suisse de coordination pour la recherche en éducation.

Allemann-Ghionda C., Lusso-Cesari V. (1986). Les échecs scolaires des enfants des travailleurs immigrés en Suisse : causes, mesures en cours d'application, perspectives. Aarau : Centre suisse de coordination pour la recherche en matière d'éducation.

Asal, H. (2014). Islamophobie : la fabrique d'un nouveau concept. État des lieux de la recherche. Sociologie, 1(5), 13-29.

Besençon, P.-A. (2015). Évolution de l'offre de formation continue destinée aux enseignants de Suisse romande. Dans O. Maulini et al. (dir.), À qui profite la formation continue des enseignants ? (p. 55-67). Paris : De Boeck Supérieur.

Bolzman, C., Fibbi, R. et Vial, M. (1996). La population âgée immigrée face à la retraite: problème social et problématique de recherche. Dans H.-R. Wicker et al. (dir.), L'altérité dans la société : migration, ethnicité, État (p. 123-142). Zurich : Seismo.

Borri-Anadon, C., Collins, T. et Boisvert, M. (2018). Pratiques d'évaluation en contexte pluriethnique et plurilingue : démarche d'accompagnement d'orthophonistes scolaires. Recherche et formation, 89, 45-56. 
Borri-Anadon, C., Potvin, M., Longpré, T., Pereira Braga, L. et Orange, V. (2018). La formation du personnel scolaire sur la diversité ethnoculturelle, religieuse et linguistique dans les universités québécoises : portrait quantitatif de l'offre de cours de deuxième cycle en éducation. Canada : Université du Québec à Montréal.

Camilleri, C. (1990). Stratégies identitaires. Paris : PUF.

Candelier, M. (2003). L'éveil aux langues à l'école primaire. Evlang : bilan d'une innovation européenne. Bruxelles : De Boeck \& Larcier s.a.

Capitanescu Benetti, A. et Mottet, G. (2020). Dilemmes et bricolages. Cahiers pédagogiques, 558, 38-39.

Dasen, P. (1991). Vers une école interculturelle, Recherches interculturelles dans l'enseignement primaire à Genève. Genève : Université de Genève Faculté de psychologie et des sciences de l'éducation Section des sciences de l'éducation.

De Rudder, V. et Vourc'h, F. (2006). Les discriminations racistes dans le monde du travail. Dans D. Fassin et E. Fassin (dir.), De la question sociale à la question raciale? Représenter la société française (p. 175-194). Paris : La Découverte.

Dhume, F. (2014). Former sur la discrimination à l'école : l'enjeu d'un travail sur et avec les processus de dénégation. Dans M. Sanchez-Mazas, N. Changkakoti et M.-A. Broyon (dir.), Éducation à la diversité. Décalages, impensés, avancées (p. 27-46). Paris : L'Harmattan.

DIP (2016). La laïcité à l'école. Genève : Département de l'instruction publique.

Frisina, F. (2017). (Faire) désapprendre l'islamophobie. Hommes et migrations, 1316, 99-107.

Gomensoro, A. et Bolzman, C. (2016). Les trajectoires éducatives de la seconde génération. Quel déterminisme des filières du secondaire I et comment certains jeunes le surmontent? Swiss Journal of Sociology, 42(2), 289-308.

Jacobs, M. (2018). Inclusion scolaire des élèves allophones ou issus de l'immigration en classe : de la difficulté à former le jugement professionnel des futurs enseignants. Recherche et formation, 89, 57-69.

Hutmacher, W. (1987). Enjeux culturels dans les politiques éducatives : une rétrospective. Dans CERI (dir.), Léducation multiculturelle (p. 356-375). Paris : OCDE.

Kerzil, J. (2002). Léducation interculturelle en France : un ensemble de pratiques évolutives au service d'enjeux complexes. Carrefours de l'éducation, 2 (14), 120-159.

Lanfranchi, A., Perregaux, C. et Thommen, B. (2000). Pour une formation des enseignantes et enseignants aux approches interculturelle. Principaux domaines de formation - Propositions pour un curriculum de formation - Indications bibliographiques. Berne : CDIP.

Larochelle-Audet, J., Borri-Anadon, C. et Potvin, M. (2016). La formation interculturelle et inclusive du personnel enseignant : conceptualisation et opérationnalisation de compétences professionnelles. Éducation et francophonie, 44 (2), $172-195$

Lorcerie, F. (1983). Enfants d'immigrés et école française : à propos du mot d'ordre de pédagogie interculturelle. Dans Larbi Talha (dir.), Maghrébins en France, émigrés ou immigrés? (p. 267-298). Paris : Editions du CNRS.

Mabilon-Bonfils, M. (2015). Lécole après Charlie. Le sujet dans la cité, 2(6), 37-47.

Mechi, A. et Sanchez-Mazas, M. (2018). Face à la discrimination en contexte scolaire : former les enseignants à la flexibilité sociocognitive. Recherche et formation, 89, 71-82.

Mottet, G. (2017a). La «cause» des descendants d'immigrés à l'école saisie par des experts et ministres de l'éducation : une catégorie d'action publique en mutation. Revue Européenne des Migrations Internationales, 33(2 et 3), $203-226$.

Mottet G. (2017b). De l'intégration des élèves immigrés à l'intégration des élèves musulmans. Une analyse à partir de deux faits divers hautement médiatisés. Hommes et migrations, 1316, 89-97.

Mottet, G. (2013). À l'«École de la diversité». Enquête sur la fabrique d'une politique éducative. Université de Genève. Thèse de doctorat (manuscrit non publié).

Mottet G. et Bolzman C. (2009). L'École et l'élève d'origine étrangère. Genèse d'une catégorie d'action publique. Genève : IES éditions. 
Ogay, T. et Edelmann, D. (2011). Penser l'interculturalité dans la formation des professionnels : l'incontournable dialectique de la différence culturelle. Dans A. Lavanchy, F. Dervin et A. Gajardo (dir.), Anthropologies de l'interculturalité (p. 4771). Paris : L'Harmattan.

Ouellet, F. (1991). L'éducation interculturelle. Essai sur le contenu de la formation des maîtres. Paris : L'Harmattan.

Perregaux, C., De Goumoëns, C., De Jeannot, D. et De Pietro, J.-F. (2003). Éducation et ourverture aux langues à l'école (EOLE). Neuchâtel : CIIP.

Perregaux, C. (1994). ODYSSEA. Accueil et approche interculturelle. Neuchâtel : COROME.

Plumelle, B. et Latour, M. (2012). La formation initiale et continue des enseignants : Allemagne, Angleterre, Finlande. Sèvres : Centre de ressources et d'ingénieries documentaires.

Potvin, M., Dhume, F., Verhoeven, M. et Ogay, T. (2018). La formation des enseignants sur la diversité et les rapports ethniques : regard comparatif France, Québec, Belgique et Suisse. Éducation et francophonie, 2(46), 30-50.

Potvin, M. et Larochelle-Audet, J. (2016). Les approches théoriques sur la diversité ethnoculturelle en éducation et les compétences essentielles du personnel scolaire. Dans M. Potvin, M.-O. Magnan et J. Larochelle-Audet (dir.), La diversité ethnoculturelle, religieuse et linguistique en éducation au Québec. Théorie et pratique (p. 109-127). Montréal : Fides Éducation.

Rey, M. (1996). D’une logique mono à une logique de l’inter. Pistes pour une éducation interculturelle et solidaire. Genève : Université de Genève, Cahiers de la section des Sciences de l'éducation.

Sanchez-Mazas, M. (2004). Racisme et xénophobie. Paris : PUF.

Sanchez-Mazas, M., Changkakoti, N. et Broyon, M.-A. (2015). Éducation à la diversité : décalages, impensés, avancées. Paris : L'Harmattan.

Sanchez-Mazas, M., Mechi, A. et Buchs, C. (2021). Des valeurs à l'expertise. Apports de la psychologie sociale à une formation des enseignants en contexte de diversité. Dans F. Lorcerie (dir.), Les fondamentaux de l'action. Rennes : Presses Universitaires de Rennes.

Santerini, M. (2002). La formation des enseignants à l'interculturel : modèles et pratiques, Carrefours de l'éducation, 2(14), 96-105.

Sayad, A. (2014) (textes rassemblés par Falaize B., Laacher, S.). L'école et les enfants de l'immigration. Paris : Le Seuil, coll. Essais critiques.

Stevens, P. et Dworkin, G. (2019). The Palgrave handbook of race and ethnic inequalities in education. New York, NY: Palgrave-Mac Millan.

Tessier, S. et Le Roy, É. (2009). Formation des professionnels à l'interculturalité. Dans S. Tessier (dir.), Familles et institutions : cultures, identités et imaginaires (p. 297-310). Paris : ERES.

Troncin, T. (2011). Pourquoi et comment ancrer la question de la diversité des élèves au cœur de la formation des enseignants? La nouvelle revue de l'adaptation et de la scolarisation, 3(55), 123-132.

Tutiaux-Guillon, N. et Verhoeven, M. (2018). Former contre les discriminations (ethno)culturelles. Recherche et formation, 89, 9-16.

Zoïa, G. (2013). Morale laïque et identité à l'école. Le Télémaque, 1(43), 73-86.

\section{Pour citer cet article}

Mottet, G. et Sanchez-Mazas, M. (2021). La formation initiale et continue des enseignant-e-s à l'interculturel : discontinuités et injonctions paradoxales. Formation et profession, 29(1), 1-18. http://dx.doi.org/10.18162/fp.2021.576 